\title{
From Frequency Domain to Temperature Domain of Transformer Liquid Insulation
}

\author{
Diego M. Robalino \\ Megger North America \\ 4271 Bronze Way \\ Dallas, TX 75237, USA
}

\author{
Raul E. Alvarez \\ IITREE - Laboratorio de Alta Tensión - FI \\ Universidad Nacional de La Plata \\ 48 y 116 La Plata, Argentina
}

\begin{abstract}
The dielectric response of the overall transformer insulation is an invaluable source of information for those involved in the manufacture, operation and maintenance of power and distribution transformers. One of the greatest advances in the use of the transformer insulation dielectric response is without a doubt the possibility of performing advanced diagnostics of the oil-paper insulation to determine the moisture concentration of the solid insulation and the quality of the liquid insulation. Moreover, based on the knowledge acquired, the dielectric response in the frequency domain allows correct definition of the thermal behavior of dielectric parameters such as power factor or dissipation factor. Dissipation factor or power factor are dependent on frequency and temperature. It is clear for field users that the thermal behavior of the dielectric parameters is related to the condition of the overall insulation system and not just to the construction or nameplate of the transformer. Therefore, the application of the dielectric response in the frequency domain is a more accurate method to determine the real thermal behavior of the power factor. In the context of this document, authors investigate different liquid insulation materials with different aging/quality conditions, their dielectric response in the frequency domain and the conversion of this response into the temperature domain.
\end{abstract}

Index terms - liquid insulation, mineral oil, dielectric response, temperature, power factor, dissipation factor

\section{INTRODUCTION}

For over 20 years, power transformer manufacturers and field operators have witnessed the evolution of different testing and condition assessment technologies. The need for accurate and reliable information, along with minimum down time for evaluation, has triggered researchers worldwide to investigate, develop and improve different non-intrusive and non-destructive methodologies to benefit the entire electrical energy industry.

To ensure the safe and reliable operation of power transformers, the analysis of the insulation system is of utmost importance and in lieu of determining the unique condition of the unit under test (UUT), dielectric response methods in time and frequency domain are widely used nowadays [1, 2]. Historically, the analysis of oil samples have given operators good understanding of the condition of the liquid insulation inside the transformer. The great advantage of this method is that it avoids the need to remove the transformer from operation. However, the limitation of this method is that samples have to be carefully taken, temperature properly recorded and containers tightly sealed to be sent to laboratories for physical or chemical analysis in accordance with international standards. In North America ASTM and IEEE standards provide the necessary guidelines to perform the oil sampling, testing and interpretation of results.

The application of transformer oil analysis together with its dielectric response in the frequency domain and in the temperature domain is investigated in this document. Advances on dielectric response measurement in the frequency domain to minimize the effect of temperature variation and testing time are used for the experimental part of this work.

\section{TRANSFORMER OIL}

\section{A. Testing Transformer Oil}

Mineral oil, mainly used together with cellulose to build the complex insulation geometry of power transformers, is non-polar and non-dispersive. In order to properly serve the purpose of insulation and cooling, mineral oil should comply with the set of properties well described in the standards. Liquid insulation sampling does not require interruption of continuous transformer operation. Therefore, a diagnostics methodology capable of interpreting the liquid insulation condition in a fast and reliable manner is always going to be a convenient resource in the field.

Physical-chemical and dielectric analysis of the liquid insulation are essential $[3,4]$. The limit requirements for transformer oil are well tabulated in [5]. A summary of the property requirements for new and service-aged mineral oil is presented in Table I. The recommended standard practices for sampling electrical insulating liquids can be found in ASTM D 923-07. Awareness of standard limits and correct testing practices derive in assertive decisions.

The neutralization number represents the total acid content in the oil. If higher than standard recommended values are observed, conductivity will increase, and so will the rate of metal corrosion of the transformer internals. Both factors shorten the life of the insulation system. Water content is also monitored and controlled to achieve adequate 